\title{
Pedal Kaposi's Sarcoma as the first sign of HIV status: A case report
}

\author{
by Sabrina Minhas, $\mathrm{DPM}^{1}$, Tracey C. Vlahovic, $\mathrm{DPM}^{2}{ }^{-}$
}

The Foot \& Ankle Journal 1 (8): 3

AIDS-Related Kaposi's sarcoma is an uncommon occurrence of the foot. Two cases of solitary pedal KS are presented of patients who had not been previously diagnosed as HIV positive prior to biopsy. Once histopathology reports returned the diagnosis of Kaposi's sarcoma, both patients received HIV tests and were found to be positive.

Key words: Kaposi’s sarcoma, AIDS, pyogenic granuloma

Published: August 2008

This is an Open Access article distributed under the terms of the Creative Commons Attribution License. It permits unrestricted use, distribution, and reproduction in any medium, provided the original work is properly cited. (The Foot \& Ankle Journal (www.faoj.org)

Kaposi's sarcoma (KS) or "multiple idiopathic hemorrhagic sarcoma" was first described in the 1800's. ${ }^{1} \quad$ Historically, this disease is known to be a rare, slowly spreading tumor confined almost entirely to older European populations. ${ }^{2}$ More recently, it has been shown to occur regularly in the African tropics as well as be associated with AIDS. $^{2}$ Kaposi's sarcoma has been found to be the most prevalent neoplastic lesion in patients who have AIDS in the United States. ${ }^{3}$

Four types of Kaposi's sarcoma exist and are caused by Human Herpesvirus 8 (HHV-8). ${ }^{2,45}$ The first type, Classic KS, is found to be prevalent in Jewish, Mediterranean, and Eastern European men and seldom seen in females.

Address correspondence to: Tracey C. Vlahovic, DPM

Associate Professor, Temple University School of Podiatric Medicine, Philadelphia, Pa.19107 email: traceyv@tample.edu

${ }^{1}$ Second year resident, Roxborough Memorial Hospital, Philadelphia, Pa. 19107.

${ }^{2}$ Associate Professor, Temple University School of Podiatric Medicine, Philadelphia, Pa. 19107.
This type occurs more commonly in patients older than fifty and presents as lower extremity lesions.

African KS, the second type, accounts for the most prevalent tumor in parts of Zaire. Even though this form may affect younger patients, it can be more aggressive by invading bone and causing various other infiltrates. The third type, AIDS-Related KS, is more common in homosexual men. ${ }^{6}$ In this type, extracutaneous growth is known to occur. The final type involves iatrogenic immunosuppresion which occurs when patients are given various antirejection medications following organ transplantation. KS and other tumors have a greater prevalence in these patients. However, once these drugs are discontinued, this form of Kaposi's sarcoma has been shown to regress. 
The typical KS lesion tends to be fairly widespread, whereas HIV related $\mathrm{KS}$ is characteristically limited to the skin and/or mucous membrances. Classic KS lesions tend to involve the oral cavity, lymphatics, lungs, trunk and extremities. ${ }^{2,5,6}$ In AIDS-Related KS, lesions on the foot and ankle are an uncommon occurrence; whereas in the Classic form, pedal lesions are more common. ${ }^{7}$ In the AIDS-Related patients, skin lesions are considered manifestation of advanced HIV disease and are more commonly seen on the superior aspects of the trunk and extremities. ${ }^{8}$ These lesions may appear as dark pink or purple to brown or black., In addition, its appearance can vary from plaques to nodules with crust. ${ }^{2,8,10}$ Various differential diagnoses exist for pedal Kaposi's sarcoma; such as pyogenic granuloma, well-differentiated angiosarcoma, amelanotic melanoma, hemangioma, purpura, lesions associated with vascular insufficiency, and ecchymoses. ${ }^{10,11}$ Interestingly, Kaposi's sarcoma lesions arise slowly and may be painless even in long-standing wounds. ${ }^{9,10}$ Essentially, most malignancies of the skin are painless. Diagnosis must be made via a skin biopsy of the questionable lesion. In the cases reviewed in this study, a solitary pedal lesion was the only indication of the patients' disease.

The exact cause of KS is still a mystery, but in AIDS-Related Kaposi's sarcoma, it has been shown that HHV-8 toxicity of the patient is related to the viral load, the CD4 count, and the HHV-8 serological activity. ${ }^{4,7}$ In rare instances, AIDS-Related KS may even be caused by trauma. ${ }^{9}$

Treatments for KS vary from simple excision to chemotherapy and radiation therapy for more extensive cases. ${ }^{7-10}$ Since each lesion of Kaposi's sarcoma evolves independently from the other, a simple excision may not prevent more from forming. ${ }^{2,710}$ So, roentgen treatments are considered favorable by many to treat and prevent further KS. ${ }^{13,14}$ HIV testing must also be performed on patients who have the lesions and have risk factors.

(C) The Foot \& Ankle Journal, 2008

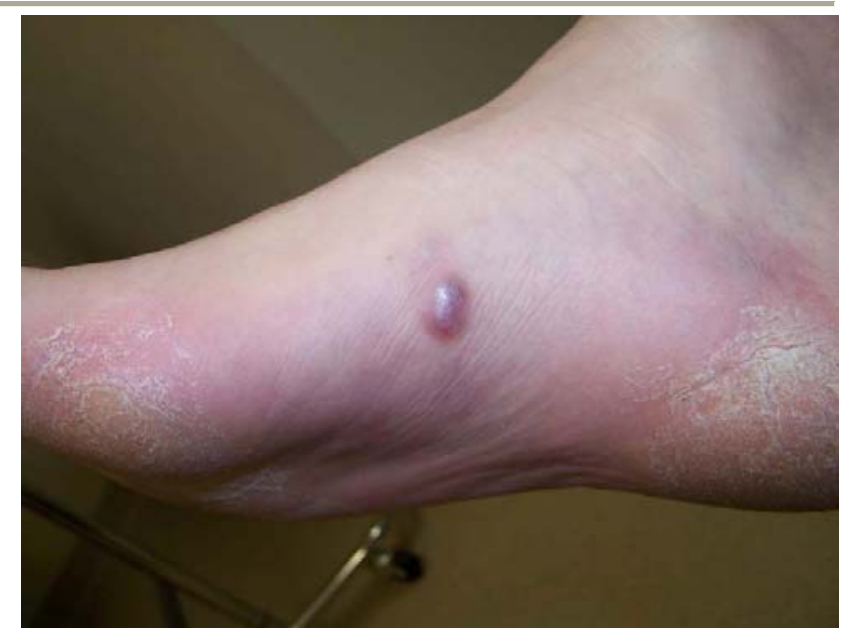

Figure 1 HIV Patient with pigmented lesion on medial aspect of foot.

The following case reports show two patients with non-painful pedal lesions as one of the first signs of HIV infection.

\section{Case 1}

A 31 year old male without a significant past medical history presents with a painful "blood blister" to the foot. Medication history included oral terbinafine for toenail onychomycosis. The patient initially complained of a painful "blood blister" that wouldn't disappear on his right foot for three months. The patient denied any trauma or new activities. He tried to "pop" the blister, but was unsuccessful in generating any serosanguinous fluid. Upon clinical examination, the lesion was a well-demarcated solitary reddishpurple nodule $(1 \mathrm{~cm} \times 1 \mathrm{~cm})$ on the medial aspect of the first metatarsal mid-shaft with no ulceration or signs of infection. (Fig. 1) It was freely mobile and appeared to be a superficial lesion. Differential diagnoses include melanoma, hemangioma, and vascular malformation. The patient requested removal by excision due to the constant rubbing of the lesion against the shoes.

An excisional biopsy was performed in the office. The pathology report returned as Kaposi's sarcoma which was strongly reactive to CD34 and negative for Smooth Muscle Antigen, Factor XIIIa and Desmin. 


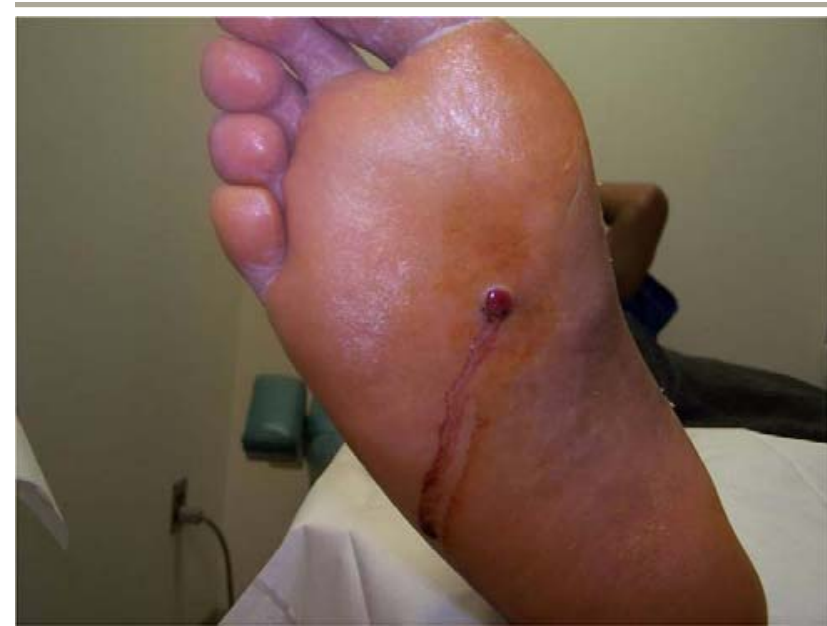

Figure 2 HIV with pyogenic granuloma-like lesion of the plantar foot.

When the patient returned to the clinic for suture removal, the diagnosis was discussed and an HIV test with a general laboratory work-up was recommended. Subsequently, the patient's HIV test was positive and the patient was then referred to the appropriate specialist. To this date, no recurrence of the lesion or of any new lesion has occurred on that foot.

\section{Case 2}

A 38 year old male presents with a solitary $0.5 \mathrm{~cm}$ x $0.5 \mathrm{~cm}$ bright red papule on the plantar surface of the right foot. The lesion was painful and sponataneously occured two months prior to presentation. Patient denied trauma to the area. Patient had no significant medical history, no known allergies to medication, and not taking any medications. The patient requested removal of the lesion. Upon examination, the lesion had the appearance of a pyogenic granuloma and was completely excised in the office. (Fig. 2)
The pathology report showed: mitotic figures, proliferation of spindle cells and slit-like vascular channels with red blood cells. The lesion was further stained for HHV-8 and a subsequent report diagnosed the lesion as Kaposi's sarcoma. During the patient's follow up visit to remove sutures, the diagnosis was discussed and an HIV test was strongly recommended.

The patient consented to HIV testing which was positive. He was referred to an HIV specialist. To this date, the foot lesion has not recurred.

\section{Discussion}

Pedal AIDS-Related KS is a rare occurrence, and even rarer for a patient's HIV status to first manifest itself as a KS lesion on the foot. Appearance of KS is considered a sign of both advanced disease and opportunistic infection. ${ }^{6}$ Young homosexual men with KS are more likely to present with AIDS-Related Kaposi's sarcoma while older heterosexual men are at higher risk to acquire Classic Kaposi's sarcoma. ${ }^{10,11}$ Exceptions exist in both cases, and a thorough history and physical exam along with laboratory tests should be performed to evaluate each individual especially post-biopsy.

When evaluating pigmented nodules and tumors on the foot, the podiatric physician should be aware of the common locations of KS lesions and include KS as a differential diagnosis along with other benign lesions. Both patients had successful excisional biopsies of their lesions, although in most cases, more aggressive therapy such as chemotherapy or radiation is warranted to eradicate the lesion. These cases demonstrate the importance of evaluating patients thoroughly post-biopsy in order to obtain an accurate diagnosis which will facilitate future treatment. 


\section{References}

1. Kaposi, M. Idiopathisches multiplex pigment sarcoma der hau ardin. F. Dermatologie (Berlina) 4:269273, 1872.

2. Elder DE, Elenitsas R, Johnson BL Jr, Murphy GF. Lever's Histopathology of the Skin. $9^{\text {th }}$ ed. Philadelphia, PA: Lippincott Williams and Wilkins, p 1229, 2005. 3. Anderson LA, Goedert JJ. Tumor markers and treatments for Kaposi sarcoma. AIDS;21:1637-1639, 2007.

4. Chang Y, Cesarman E, Pessin MS, Lee F, Culpepper J, Knowles DM, et al. Identification of herpesvirus-like DNA sequences in AIDS-associated Kaposi's sarcoma. Science;266:1865-1869, 1994.

5. Martino RJ, Pulse C, Zegarelli DJ. Classic Kaposi’s sarcoma presenting in the oral cavity: a case report and literature review. Columbia Dental Review 1996.

6. Dezube BJ, Groopman JE. AIDS-related Kaposi's sarcoma: epidemiology and pathogenesis. Hematology/Oncology Clinics of North America;10:1023-1029, 1996.

7. Montes C, Luepschen OM. Kaposi's sarcoma of the foot in the HIV patient. The Journal of Foot and Ankle Surgery;33:341-345, 1994.

8. Cohen EJ, Cole D, Stewart DM, Weiss G, Kosinski M, Giorgini R. Kaposi's sarcoma of the lower extremity as the first sign of AIDS. Journal of the American Podiatry Medical Association;80:127-134, 1990.

9. Berkowitz KD, Bonner AC, Makimaa B, Flash JP, Sasken H, Blaise JF. Trauma-induced Kaposi's sarcoma of the hallux. An unusual case. Journal of the American Podiatric Medical Association;88:500-505, 1998.

10. Berlin SJ, Jurd JA. Kaposi's sarcoma in the foot. Clinics in Podiatric Medicine and Surgery;9:849-855, 1992.

11. Cangialosi CP, Schnall SJ. Kaposi's sarcoma of the foot: a case report. Journal of the American Podiatry Association;66:525-527, 1976. 12. Tedeschi R, Enbom M, Bidoli E, Linde A, De Paoli $P$, Dillner J. Viral load of human herpesvirus 8 in peripheral blood of human immunodeficiency virusinfected patients with kaposi's sarcoma. Journal of Clinical Microbiology; 39:4269-4273, 2001.

13. Mazzanti JA, Hugar DW. Kaposi's sarcoma-an overview. The Journal of Foot Surgery;19:71-73, 1980. 14. Levi MJ. Classic Kaposi's sarcoma. Journal of the Podiatric Medical Association; 95:586-588, 2005. 